\title{
La formación ciudadana en biblioteconomía y documentación como experiencia docente innovadora ${ }^{1}$
}

\author{
Pedro López López \\ Inmaculada Vellosillo González ${ }^{* *}$
}

\section{Resumen}

Se defiende la necesidad de atender la dimensión de ciudadanía en la formación en Biblioteconomía y Documentación. Se exponen los argumentos que justifican esta formación y se citan recomendaciones y otros textos de organismos internacionales como UNESCO, Consejo de Europa e IFLA. Por otro lado, se justifica la consideración de este tipo de formación como un elemento de innovación y calidad docente en una titulación que requiere compromiso cívico y defensa y promoción de los valores democráticos. Por último, se rechaza la orientación excesivamente mercantilista que las autoridades educativas y muchos profesores están aceptando para construir el Espacio Europeo de Educación Superior. No obstante, esta orientación puede ser corregida desde la toma de conciencia de la comunidad docente.

Palabras-clave: La formación del bibliotecario. La inclusión social. Ciudadanía.

Título: A educação cívica na biblioteca e da ciência da informação como pedagógicas inovadoras

\section{Resumo}

Defende-se a necessidade de abordar a dimensão da cidadania na formação em biblioteconomia e documentação. São expostos os argumentos que justificam esta formação e cita-se as recomendações e outros textos de organismos internacionais como a UNESCO, Conselho da Europa e IFLA. Por outro lado, justifica-se a consideração de que este tipo de formação como um elemento de inovação e qualidade no ensino de uma formação requer participação cívica e defesa dos valores democráticos. Por último, rejeita-se a orientação excessivamente mercantilista que muitos professores e autoridades educativas estão aceitando para a construção do Espaço

\footnotetext{
${ }^{1}$ Comunicación presentada en el III Encuentro Ibérico de docentes e investigadores en Información y Documentación (Salamanca, 5-7 mayo 2008)

* Profesor Titular de la Facultad de Ciencias de la Documentación, Universidad Complutense de Madrid. pedrolopez@ccdoc.ucm.es

Profesora Titular de la Facultad de Ciencias de la Documentación, Universidad Complutense de Madrid. inma@ccdoc.ucm.es
} 
Europeu do Ensino Superior. No entanto, esta orientação pode ser corrigida a partir da sensibilização da comunidade educativa.

Palabras-clave: Formação do bibliotecário. Inclusão social. Cidadania.

Title: Civic education in library and information science as innovative teaching

\begin{abstract}
The need to emphasize citizenship within Library and Information Science education is stressed. Justification for improving this need is presented, with mention to recommendations made by international organizations such as UNESCO, the Council of Europe, and IFLA. This kind of education is also considered a key element in quality teaching and innovation, given the fact that LIS graduates will need to defend democratic values and become socially committed during their professional careers. Also, the idea of constructing a market-oriented European Space for Higher Education, as defended by education authorities and some faculty is questioned. It is argued that this market-oriented approach may be changed if the faculty become conscious and oppose it.
\end{abstract}

Keywords: Training of the librarian. Social inclusion. Citizenship.

Decía Fernando Savater en un artículo reciente que "...la sociedad progresa cuando amplía y consolida las capacidades de la ciudadanía" ${ }^{2}$.

Casi siempre que se habla de innovación parece que entendemos que esta idea va ligada casi exclusivamente a la dimensión tecnológica, de tal manera que parece necesario disponer de abundantes medios audiovisuales para explicar cualquier materia, ya sea Química Orgánica, Historia de los Griegos, Macroeconomía o Metafísica.

Sin embargo, la comunicación que presentamos para defender la necesidad de una formación ciudadana en la titulación de Biblioteconomía y Documentación, es una propuesta que se encuadra con toda propiedad en el marco de la innovación docente. Algunas razones que nos llevan a esta consideración son las siguientes:

- En junio de 2005 el secretario de Estado de Universidades declaraba (III Jornadas de Redes de Investigación en Docencia Universitaria, Universidad de Alicante) que "La Universidad del futuro tiene como misión formar a ciudadanos".

\footnotetext{
2 "Regreso al progreso", diario El País, 2-8-07.
} 
La formación ciudadana en biblioteconomía y documentación como experiencia docente innovadora

- En el marco de la política educativa del Consejo de Europa, la educación para la ciudadanía democrática es señalada como un factor de innovación docente dentro de los métodos de enseñanza y de los planes de estudio (recomendación [Rec(2002)12] ) del Comité de Ministros de Educación. La declaración del 2005 como Año Europeo de la Ciudadanía a través de la Educación aclaraba que esta declaración no se limitaba a una campaña puntual, sino que tal año era un "punto de partida de un proceso que deberá continuar los próximos años”.

- El programa de Educación para la Ciudadanía de la Generalitat de Catalunya es considerado de innovación educativa.

- El artículo 3.2.e. de los estatutos de la Universidad Complutense (2003) señala que una de sus funciones es "la formación en valores ciudadanos de los miembros de la comunidad universitaria”.

- El año 2005, el Vicerrectorado de Innovación y Espacio Europeo de Educación Superior aprobó, en su convocatoria de proyectos de innovación y mejora de la calidad docente, el proyecto de investigación La educación en valores y el Espacio Europeo de Educación Superior. Estrategias docentes en Biblioteconomía y Documentación. El proyecto se ocupaba de la formación en valores democráticos.

Esta pequeña muestra de referencias indica que la formación ciudadana es considerada por un amplio número de instituciones un factor de calidad y de innovación docente. El proyecto de investigación que los autores de este trabajo desarrollaron en el marco de la convocatoria de proyectos de innovación de la Universidad Complutense 2005, ha dado lugar a un conjunto fructífero de actuaciones: cuatro comunicaciones a congresos, publicación del libro "Educación para la Ciudadanía y Biblioteca Escolar" (en prensa), participación en la III Jornada de Innovación Educativa en la Universidad Complutense (6-6-06), celebración del Seminario "Didáctica de los valores democráticos en Biblioteconomía, Documentación y Periodismo" (ICE Univ. Complutense, 20-21 octubre 2006), implantación del módulo "Biblioteca y Sociedad” en el Master en Gestión de la Documentación y Bibliotecas de nuestra facultad, e implantación de la asignatura de libre configuración "Derechos Humanos, Ciudadanía y Sociedad de la Información" (desde el curso 2005-2006), asignatura que ha sobrepasado con creces las expectativas iniciales de matriculación. 
El hecho de que el debate educativo sobre educación ciudadana se haya centrado en los niveles preuniversitarios puede hacer pensar que está fuera de lugar en la Universidad, adonde se supone que el individuo llega suficientemente formado en estos aspectos. Es curioso hasta qué punto la Universidad no se ha dado por aludida. Hay razones suficientes para defender la necesidad de esta formación en la etapa universitaria. Cuando la Constitución Española, en su artículo 27.2 (inspirado en el 26 de la Declaración Universal de Derechos Humanos) dice que "la educación tendrá por objeto el pleno desarrollo de la personalidad en el respeto a los principios democráticos de convivencia y a los derechos y libertades fundamentales”, no se refiere a ningún nivel educativo concreto, por lo que no tiene sentido entender que la etapa universitaria queda excluido. De hecho, el artículo el artículo 3.2.e. de los estatutos de la Universidad Complutense señala que una de sus funciones es "la formación en valores ciudadanos de los miembros de la comunidad universitaria”. Igualmente, el artículo 3.2.b de los estatutos de la Universidad de Barcelona declara que entre sus objetivos están "el fomento del pensamiento crítico y de la cultura de la libertad y el pluralismo, y la transmisión de los valores cívicos y sociales de una sociedad democrática”. Sin embargo, más allá de fraseología y de actos puntuales, puede afirmarse que no existe una verdadera política educativa al respecto. Hay que pensar que estas palabras quedan en un mero "brindis al sol" si no se reflejan en planes concretos de actuación.

Naturalmente, nuestro convencimiento de la necesidad de formar en ciudadanía a los estudiantes de Biblioteconomía y Documentación no es un empeño caprichoso ni una ocurrencia que ha partido de la nada. Antes de mencionar otros textos de referencia, destacaremos las razones por las que creemos que cualquier estudiante universitario debe recibir esta formación:

- La formación ciudadana prepara para un ejercicio socialmente responsable de la futura profesión, y no de mero lucro personal.

- Diversos textos de referencia de organismos como el Consejo de Europa o la Unesco aluden a la conveniencia de formar ciudadanos en la universidad A continuación señalaremos algunos.

- En la edad universitaria, los jóvenes comienzan a asumir responsabilidades adultas para con la comunidad.

- El individuo se enfrenta por primera vez a unas elecciones para elegir el partido gobernante. 
La formación ciudadana en biblioteconomía y documentación como experiencia docente innovadora

- La inclusión del área de Educación para la Ciudadanía y los Derechos Humanos en la Ley Orgánica de Educación afecta a los universitarios de cualquier titulación, habida cuenta de que los futuros profesores de educación secundaria y bachillerato salen de todas las carreras universitarias.

En cuanto a la formación inicial de los bibliotecarios, instituciones como IFLA y UNESCO destacan el papel de las bibliotecas en la promoción de los valores democráticos.

Citamos a continuación algunos textos en relación con lo que venimos diciendo:

\section{Formación universitaria}

Parece de obligada referencia es la Declaración Mundial sobre la Educación Superior en el Siglo XXI: Visión y Acción (UNESCO, octubre 1998), que hace repetidas alusiones a la dimensión ciudadana de la universidad en el primer artículo, declarando que entre sus misiones y funciones están:

1.a) "Formar diplomados altamente cualificados y ciudadanos responsables..."; b) "Constituir un espacio abierto para la formación superior que propicie el aprendizaje permanente... así como oportunidades de realización individual y movilidad social con el fin de formar ciudadanos que participen activamente en la sociedad..."; c.) "Contribuir a proteger y consolidar los valores de la sociedad, velando por inculcar en los jóvenes los valores en que reposa la ciudadanía democrática...”

Más adelante, el artículo 9.b declara que “las instituciones de educación superior deben formar a los estudiantes para que se conviertan en ciudadanos bien informados y profundamente motivados, provistos de un sentido crítico y capaces de analizar los problemas de la sociedad, buscar soluciones para los que se planteen en la sociedad, aplicar éstas y asumir responsabilidades sociales”. Por último, el artículo 14.b declara la obligación de la UNESCO de "poner de relieve la función de la enseñanza superior para la educación cívica, el desarrollo sostenible y la paz”.

Otra referencia de interés es la Recomendación relativa a la Condición del Personal Docente de la Enseñanza Superior (UNESCO, 1997). Entre sus principios rectores, destaca que “los objetivos de paz, cooperación, desarrollo sostenible, etc. que persiguen los Estados miembros de las Naciones Unidas "requieren, entre otras cosas, una educación para la paz y en 
la cultura de paz [...] así como diplomados de instituciones de enseñanza superior calificados y cultos, capaces de atender a la comunidad como ciudadanos responsables...”.

También merece mención la declaración formulada por el Consejo de Europa en Estrasburgo en junio de 2006 bajo el título Responsabilidad de la Educación Superior para una cultura democrática: ciudadanía, derechos humanos y sostenibilidad. En ella se precisa el concepto de cultura democrática: "valores democráticos, capacidad de reflexión y de acción, juicios éticos, calidad de análisis y capacidades de compromiso". Asimismo, se declara la convicción de que "la enseñanza superior juega un papel esencial en la transmisión de la cultura democrática" y la responsabilidad de "enseñar a cada generación a renovar y a desarrollar las actitudes, valores y competencias necesarias para traducir este compromiso en hechos" y de promover una cultura democrática, uno de cuyos principios, se señala, es una ciudadanía democrática activa.

En lo que nos ocupa, la política educativa del Consejo de Europa se ha concretado en los últimos años en la declaración del año 2005 como "Año Europeo de la Ciudadanía a través de la Educación". El documento de orientación elaborado para esta actuación, consultable en la página web del Ministerio de Educación y Ciencia ${ }^{3}$, deja claro que no se trata de una "campaña" que termina con el año 2005, sino de un "punto de partida de un proceso que deberá continuar los próximos años". El objetivo del "Año", se dice, "es estimular un interés general por la educación para la ciudadanía democrática". Asimismo, en el documento se alude al hecho de que la Educación para la Ciudadanía (EDC) y la Educación para los Derechos Humanos (EDH) han sido prioritarias para el Consejo de Europa desde el año 1997, inspirando diversos encuentros y documentos. Uno de los más destacados es la Recomendación Rec (2002) del Comité de Ministros a los Estados miembros sobre la educación para la ciudadanía democrática, que destaca que ésta "debería estar en el centro de las reformas y de la praxis de las políticas educativas", así como que "es un factor de innovación para el conjunto de la organización y gestión de los sistemas educativos, así como de los programas y de los métodos pedagógicos". Por último, al concretar los objetivos, se señala la necesidad de habilitar a quienes toman las decisiones y a los profesionales de todos los niveles para crear y desarrollar programas duraderos en materia de educación para la ciudadanía y educación para los derechos humanos.

\footnotetext{
${ }^{3}$ http://www.educacionciudadania.mec.es/documentos.html
} 
La formación ciudadana en biblioteconomía y documentación como experiencia docente innovadora

Antes de terminar este apartado, conviene dejar constancia de que entre la cultura política de la ciudadanía deben figurar los derechos humanos como un elemento de primer orden, ya que aportan una dimensión sustantiva a la democracia. La ciudadanía debe saber en qué consiste la democracia, qué instituciones la defienden, qué es ser ciudadano y qué son los derechos humanos, así como qué instrumentos los protegen. Existen importantes documentos que aconsejan la educación en derechos humanos y que representan un compromiso para el Estado. Así, la Resolución 534/144 de la Asamblea General de las Naciones Unidas, de 9 de diciembre de 1998, dice que "incumbe al Estado la responsabilidad de promover y facilitar la enseñanza de los derechos humanos y las libertades fundamentales en todos los niveles de la educación”. Otros documentos relevantes insisten en la necesidad de esta formación: Programa Mundial para la EDH, 2005; Declaración de la Conferencia Mundial de Derechos Humanos, 1993; Recomendación R(85) 7 del Consejo de Europa, Declaración de la 44 Conferencia Internacional de Educación (Ginebra, 1994). En nuestro país, muy recientemente, la Ley 27/2005, de 30 de noviembre, de fomento de la educación y la cultura de la paz (BOE 1-12-05), en su artículo 2.6 establece que corresponde al Gobierno "promover un incremento del conocimiento público y de la enseñanza del Derecho Internacional humanitario y de la legislación sobre Derechos Humanos”. A todas luces, la Universidad es un ámbito idóneo para llevar a cabo ese mandato.

Queda claro que la promoción de la democracia, la ciudadanía y los derechos humanos es una obligación del sistema educativo de cualquier país en todos los niveles. Evidentemente, "ciudadanía" es un concepto político, y se equivocan rotundamente los que apuestan por una formación exclusivamente técnica y pretendidamente "apolítica".

Que estos textos sean tomados por mera "palabrería" o que los docentes prestemos atención a la responsabilidad que nos marcan, parece que depende, desgraciadamente, del voluntarismo de cada uno.

\section{El Espacio Europeo de Educación Superior}

El Espacio Europeo de Educación Superior se ha convertido en una coartada para adaptar más ajustadamente la universidad al mundo de la empresa. No es ésta una percepción que parta exclusivamente de posiciones críticas, sino que los propios documentos generados desde el 
proyecto piloto Tuning así lo reconocen. El lenguaje de las competencias que se ha impuesto no procede del mundo de la educación, sino del empresarial, que, partiendo del moldeamiento de valores que se ajusta a los intereses de los sectores punteros de la economía (especialmente tecnología, finanzas mundializadas y grandes empresas de servicios), se ha convertido en la referencia para seleccionar "las habilidades que debe proporcionar el sistema educativo" (Sennett, 2006).

En la "Carta abierta a los profesores de Universidad" redactada por el colectivo denominado "Profesores por el Conocimiento" y firmada por unos 2.500 profesores e investigadores universitarios, se denuncia que

"entre las competencias genéricas o transversales (de especial importancia en los estudios de grado) hallamos aquellas que fijan los departamentos de recursos humanos de las empresas, tales como motivación de logro, espíritu emprendedor, liderazgo, y un largo etcétera. Ello supone que las universidades deberán modificar su oferta para dar cabida, no sólo a la formación teórica y su aplicación práctica en derecho, medicina, ingeniería, etc., sino que los profesores habrán de ir moldeando a los futuros asalariados conforme a los criterios de sus eventuales empleadores".

El concepto de sociedad es bastante más amplio que el de mercado, y naturalmente es inaceptable tomar la "sociedad civil" por el mundo empresarial, como algunos parecen entender. Y esta identificación conduce a resultados nada deseables en el campo de los servicios públicos. El profesor Albatch, del Boston Collage (cit. por Moles Plaza, 2006, p. 169), habla del colapso del bien común en el ámbito educativo y en un marco más general, con una excelente descripción del proceso de mercantilización:

"En la educación está ocurriendo una revolución. La educación se está transformando en
un bien de consumo comercializado internacionalmente. Ya no es vista primordialmente
como un conjunto de habilidades, actitudes y valores necesarios para el fortalecimiento
de la ciudadanía y para la efectiva participación en la sociedad moderna, o sea, como una
contribución clave al bien común de cualquier sociedad. En lugar de esto se ve cada vez
más, como un bien de consumo que puede ser comprado por un consumidor para
adquirir un conjunto de habilidades que serán utilizadas en el mercado; o es vista como
un producto que puede ser comprado o vendido por corporaciones multinacionales,
instituciones académicas convertidas en negocios o por otros proveedores".

Insistir en una capacitación exclusivamente técnica, sin amplitud de miras y que eluda el sentido integral de la educación es, por un lado, rebajar las titulaciones universitarias a mera formación profesional, y, por otro lado, favorecer un tipo de profesional desvinculado del 
La formación ciudadana en biblioteconomía y documentación como experiencia docente innovadora

compromiso social; es producir "idiotas morales" enmarcados en el "cretinismo profesional" del que hablaba Marx, seguramente competitivos en aspectos muy especializados, pero muy alejados de lo que requiere un perfil profesional completo.

Si bien las autoridades ministeriales y rectorales han cedido en buena medida a las presiones del mercado considerando que éste es el que debe marcar en exclusiva las pautas de las transformaciones en los planes de estudio, todavía la comunidad universitaria puede corregir algo de este sesgo de excesivo mercantilismo con que se está construyendo el EEES. Una forma de hacerlo es potenciando competencias como Compromiso Ético (véase, por ejemplo, Traver y García, 2006) y reclamando atención para documentos como los citados anteriormente.

\section{Textos del contexto bibliotecario}

Completemos esta comunicación con otras referencias del mundo propiamente bibliotecario que vienen a reforzar las ideas que estamos defendiendo.

- La Declaración de Caracas sobre la Biblioteca Pública como factor de desarrollo e instrumento de cambio en América Latina y el Caribe (1985) proclama que la biblioteca pública debe estimular la participación de la población en la vida nacional, "incrementando así el papel de la Biblioteca como instrumento facilitador de cambio social y de participación en la vida democrática”. Asimismo, afirma que la biblioteca pública debe "promover la formación de un lector crítico, selectivo y creativo... capacitando así a cada individuo para jugar un papel activo en la sociedad”. Capacitar para el desempeño de un papel activo en la sociedad no puede significar otra cosa que formar ciudadanos proactivos.

- El Manifiesto IFLA/UNESCO a favor de las Bibliotecas Públicas (1994) proclama que “la libertad, la prosperidad y el desarrollo de la sociedad y de la persona son valores fundamentales que sólo podrán alcanzarse si ciudadanos bien informados pueden ejercer sus derechos democráticos y desempeñar un papel activo dentro de la sociedad. La participación constructiva y la consolidación de la democracia dependen de una buena educación y de un acceso libre e ilimitado al conocimiento, el pensamiento, la cultura y la educación.” 
- La Resolución del Parlamento Europeo sobre el papel de las bibliotecas en la sociedad moderna (1998) reconoce como una de las tareas básicas de las bibliotecas públicas “ofrecer a la ciudadanía activa una información básica imprescindible al alcance de todos... de esta forma, contribuyen a construir una sociedad de la información democrática, abierta y transparente”. Asimismo, reconoce a la biblioteca como un instrumento para la puesta en práctica de la ciudadanía activa mencionada en el Tratado de Ámsterdam.

- La Declaración de la IFLA sobre las bibliotecas y la libertad intelectual (1999) afirma que "las bibliotecas contribuyen al desarrollo y mantenimiento de la libertad intelectual y ayudan a salvaguardar los valores democráticos básicos y los derechos civiles universales".

- La Declaración del Comité de Libre Acceso a la Información y a la Libertad de Expresión de la IFLA (1999) señala que las bibliotecas "ayudan a preservar los valores democráticos básicos y los derechos civiles universales”.

- El Manifiesto de la Biblioteca Escolar (UNESCO/IFLA, 1999) señala entre las funciones de este tipo de biblioteca "proclamar la idea de que la libertad intelectual y el acceso a la información son indispensables para adquirir una ciudadanía responsable y participativa en una democracia”.

- Las Pautas del Consejo de Europa y EBLIDA (2000) sobre legislación y política bibliotecaria en Europa señalan la importancia de las bibliotecas en la democratización de los Estados, así como la necesidad de dedicar un esfuerzo conjunto a la formulación de una serie de principios acordes con los valores democráticos y compatibles con los principios constitucionales de los Estados miembros, que puedan inspirar la elaboración de sus políticas en materia de legislación y política de bibliotecas”. Estas políticas, se dice, deben "destacar el papel de los servicios bibliotecarios como factor necesario para el mantenimiento y el desarrollo de la democracia”.

- Las directrices IFLA/UNESCO para el desarrollo del servicio de bibliotecas públicas (2001), al hablar de la finalidad de la biblioteca pública (punto 1,3), dice que las bibliotecas "desempeñan un importante papel en el progreso y el mantenimiento de una sociedad democrática...”. Igualmente, en el punto 1.4 se afirma que la biblioteca pública 
La formación ciudadana en biblioteconomía y documentación como experiencia docente innovadora

"contribuye a la creación y el mantenimiento de una sociedad bien informada y democrática...”. Al hablar de redes electrónicas (punto 3.8), las directrices atribuyen a las bibliotecas públicas la función de instrumentos para la igualdad de oportunidades que “deben posibilitar el acceso de todos los ciudadanos a la información para que puedan así desenvolverse a escala local, adquirir datos esenciales sobre el proceso democrático y participar de forma positiva en una sociedad cada vez más global”. Evidentemente, la idea de participación en la sociedad está en el corazón del concepto de ciudadanía.

- El documento "Valores éticos compartidos por las bibliotecas nacionales (resolución aprobada en la XV Asamblea General de ABINIA, Chile, 2004) destaca entre estos valores "la promoción de una cultura democrática, de libertad y participación ciudadana”, así como "la promoción de los derechos"universales de los pueblos a la educación, la cultura y los conocimientos", derechos humanos directamente relacionados con la actividad bibliotecaria.

- La Declaración de Buenos Aires sobre Información, Documentación y Bibliotecas, proclamada en el marco del Primer Foro Social de Información, Documentación y Bibliotecas (Buenos Aires, 2004) destaca en diversos puntos el papel de las bibliotecas y demás centros de información en la promoción de los valores democráticos, el impulso de la práctica de la democracia, la contribución al desarrollo de los derechos humanos y el compromiso social de los bibliotecarios con la paz.

- El plan estratégico 2004-2005 del Programa de Acción para el Desarrollo a través de las Bibliotecas (IFLA) dice que las bibliotecas "deben proporcionar a todo el mundo la oportunidad de participar en los procesos democráticos de la sociedad”.

- El Manifiesto de Alejandría (2005) afirma que las bibliotecas y los servicios de información son "vitales para una Sociedad de la Información abierta y democrática. Las bibliotecas son esenciales para una ciudadanía bien informada y un gobierno transparente, así como para la implantación del gobierno electrónico”.

\section{Conclusiones}

Estas y otras referencias del ámbito bibliotecario, que relacionan la actividad bibliotecaria con la promoción de la democracia, la ciudadanía y los derechos humanos, añaden argumentos a 
los ya señalados en los textos referentes a la educación superior. Las profesiones de bibliotecario, documentalista $\mathrm{y}$ archivero ejercen una función social que reclama compromiso cívico (Rodríguez Parada y Ferran Vives, 2007). Una función que deriva del papel que los derechos de acceso a la información, el conocimiento, la cultura y la educación cumplen para la construcción de la sociedad democrática.

Concluyendo, defendemos que a un estudiante de Biblioteconomía y Documentación no sólo deben enseñársele capacidades operativas, sino que también debe aportársele un bagaje cultural que le permita, además de ejecutar tareas, pensar críticamente en la función social de su profesión, en los derechos ciudadanos que ésta permite satisfacer a la colectividad, en el concepto de servicio público -tan vinculado a la biblioteca pública-, en qué aportan las bibliotecas y los archivos a la democracia, y en cómo colaborar con la democratización del acceso a la cultura y al conocimiento científico.

Estos razonamientos se enmarcan en la premisa que defiende el director del Instituto de Ciencias de la Educación de la Universidad de Barcelona, Miquel Martínez: "no puede entenderse una formación universitaria de calidad que no incorpore en forma sistemática y rigurosa situaciones de aprendizaje ético y de formación ciudadana” (Martínez, 2006).

\section{Referencias bibliográficas}

Martínez, Miquel. Formación ciudadana y educación superior. Revista Iberoamericana de Educación, no 42, 2006, pp. 85-102.

Moles Plaza, Ramón J. ¿Universidad S.A.? Público y Privado en la educación superior. Barcelona: Ariel, 2006.

Rodríguez Parada, Concepción; Ferran Vives, Josep. Reflexions a l'entorn de la deontologia dels bibliotecaris-documentalistes. En: Col-legi Oficial de Bibliotecaris-Documentalistes de Catalunya. Bibliodoc: anuari de biblioteconomia, documentació i informaci 2006. Barcelona: Col-legi Oficial de Bibliotecaris-Documentalistes, 2007.

Sennett, Richard. La cultura del nuevo capitalismo. Barcelona: Anagrama, 2006. 
La formación ciudadana en biblioteconomía y documentación como experiencia docente innovadora

Traver Martí, Joan Andrés y García López, Rafaela. La técnica puzle de Aronson como herramienta para desarrollar la competencia "compromiso ético" y la solidaridad en la enseñanza universitaria. Revista Iberoamericana de Educación, no 40/4, 2006, pp. 1-9. 